\title{
SESSION 3: Sugar transport
}

\section{Regulation of Facilitated Hexose Transporter Activity}

\section{Chris Cheeseman}

Department of Physiology, University of Alberta, Canada

Several members of the facilitated hexose transporter family (SLC2A) are expressed in the mammalian small intestine and their activity can be regulated by a series of different mechanisms to ensure the appropriate handling of their various substrates. GLUT5 has been demonstrated to be key in the absorption of fructose, being expressed in the apical membrane of the duodenal and jejunal enterocytes, and in humans also in the basolateral membrane (BLM). GLUT2 has long been known to be expressed in the basolateral membrane and to mediate the exit of glucose and fructose from the epithelium. The activity of GLUT2 in the BLM can be regulated by the release of enteric peptides such as GLP2 and this appears to result from a change in transporter activity rather abundance in the membrane. More recently, evidence has been presented for a second possible role of GLUT2 as a highly regulated route of entry for glucose, and possibly fructose, via the transient insertion of the protein into the brush-border membrane (BBM). Currently, it is not known how this rapid insertion and subsequent removal is achieved at the molecular level, but signaling does seem to involve a combination of sweet taste receptors, SGLT1 activity and the release of GLP2 into the circulation.

Recently two additional members of the GLUT family have been shown to be expressed in the mammalian small intestine, GLUT7 and GLUT9. These are class II members of the SLC2A family and while GLUT5's function has been well defined much less is known about the others. GLUT7 appears to be a high affinity, low capacity glucose and fructose transporter as was originally believed for GLUT9. However, a series of genome scanning studies reported in 2009 indicated a role for GLUT9 in the handling the organic anion urate. We have subsequently determined that the transport of urate is electrogenic and does not involve the coupling with any other ions. GLUT9 is a urate exchanger but the driving force for the substrate's net movement is the electrochemical protential which will depend upon the urate concentration across the membrane and the membrane potential. Thus, it is proposed that in some tissues GLUT9 functions primarily as a urate effluxer, promoting the substrate movement either into the blood stream or tissue lumen. In the human alternate splicing results in the expression of two GLUT9 isoforms, 9a (or full length) and 9b (or $\Delta \mathrm{N})$. The latter has a significantly truncated $\mathrm{N}$ terminus, which differs significantly in sequence from that of the full length isoform. Surprisingly, glucose and fructose do not compete with urate for GLUT9 mediated fluxes, but in the 9a isoform they can exchange for urate and so extracellular hexose could help promote urate efflux from the cell. 
Comparison of the N-termini of the two GLUT9 isoforms shows that there are serine residues which may be PKA or PKC phosphorylation sites and that these differ between the two proteins. Using the oocyte expression system we have examined the ability of the PKA and PKC signaling systems to change the activity of GLUT9 and found that PKC changes the kinetics of basolateral GLUT9a, while PKA appears to regulate GLUT9b activity in the BBM. Data to support this contention comes from experiments in which PMA or forskolin were added to oocytes expressing GLUT9a or 9b results prior to measuring urate transport kinetics. These altered fluxes do not result from changes in protein abundance in the membrane as determined by biotinylation and Western blotting of isolated plasma membranes. Flux studies of urate uptake into isolated rings of rat colon will also be discussed.

Thus, it is likely that urate movement across epithelia such as the small intestine, colon and kidney can be regulated to promote either absorption or secretion. 


\section{Pathological accumulation of GLUT2 in enterocytes of morbidly obese subjects consuming unbalanced high fat diets}

Milena Monteiro-Sepulveda ${ }^{1}$, Kevin Garbin ${ }^{1}$, Christine Poitou ${ }^{2,3}$, Aurélie Cotillard ${ }^{2}$, Jules Gilet $^{1}$, Amélie Lacombe ${ }^{6}$, Joan Tordjman ${ }^{2}$, , Christophe Magnan ${ }^{6}$, Karine Clément ${ }^{2,3}$, Armelle Leturque $^{1}$ and Edith Brot-Laroche ${ }^{1}$

Centre de Recherche des Cordeliers, 15 rue de l'Ecole de Médecine, ${ }^{1}$ Team 9, ${ }^{2}$ Team 7.

Introduction: Sugar absorption relies on the coordinated functions of SGLT1, GLUT2 and GLUT5 transporters in enterocyte plasma membranes. In rodents, a regulated trafficking of GLUT2 from internal stores to apical membranes modulates sugar uptake into enterocytes in response to a sugar rich meal. Intracellular GLUT2 compartments remain to be identified.

Aim: This study aimed to test the hypothesis that intracellular GLUT2 locations in enterocytes of morbidly obese human subjects is altered by their eating unbalanced high fat diet.

Methods : Obese subjects $(n=62)$ from a gastric surgery program were recruited in Hôtel-Dieu Paris, France. Subjects were aged 19-64 years with BMI $\geq 40 \mathrm{~kg} \cdot \mathrm{m}^{-2}$ and their eating behaviour recorded by a dietician. GLUT2 subcellular locations were analysed in a jejunal tissue bank usually discarded during bypass or in jejunal biopsies in 7 lean subjects. Statistical correlations with phenotype of obese subjects were established. Model mice were fed a high-fat/low-carbohydrate diet for a year. Subcellular GLUT2 location was analyzed by immunofluorescence in sections and by confocal microscopy (LSM710 Zeiss Germany) using tailor made GLUT2 antibodies targeting C-terminus (Eurogentec, France) and extracellular loop 1 (Gifts from Gould, Glasgow \& Kellett, York). Antibodies to intracellular membrane markers were Lamp1 (late endosome / lysosome), BIP/GPR78 and PDI (ER). MGAT4a (Golgi Glycosyl transferase IV) was used to address glycosilation issues in obese subjects.

Results : In 39\% human obese subjects, GLUT2 accumulated above enterocyte nuclei and 71\% exhibited also apical GLUT2. This intracellular accumulation of GLUT2 colocalized with EEA1, but not BIP, PDI or LAMP1. EEA1/GLUT2 accumulation above nuclei revealed link with high-fat lowcarbohydrate diet of subjects but not with calorie intake. MGAT4a, a key element in GLUT2 trafficking, was reduced 2 fold as compared to apical GLUT2. In mice fed HFLC diet, GLUT2 was also sequestered above nuclei and glucose absorption was decreased.

Conclusion. All together our results in human obese subjects and mice show that GLUT2 is sequestered in intracellular compartments above enterocyte nuclei in response to unbalanced fat diet. In mice, intestinal absorption was reduced. High fat consumption and lower glycosilation capacities trigger impaired GLUT2 trafficking in enterocytes and affect intestinal function. 


\title{
Heterogeneity in electrogenic glucose transport features in the porcine jejunum and ileum
}

\author{
Jens Herrmann, Bernd Schröder, Gerhard Breves \\ Department of Physiology, University of Veterinary Medicine Hannover, Foundation, Bischofsholer Damm 15, 30173 \\ Hannover (Germany)
}

Introduction: Although it has been shown in several species that the highest expression levels of SGLT1 are in the jejunum, recent findings indicated that, at least in pigs, the distal part of the small intestines, the ileum, seems to absorb glucose with higher efficiency than the jejunum.

Aim: To comparatively evaluate the basal efficiency of glucose absorption and its respective dynamics in the porcine jejunum and ileum by electrophysiological methods.

Methods: 14 male weaned pigs were fed with standard chow for 1 week until slaughtering. $80 \mathrm{~cm}$ segments were taken from the jejunum and ileum. Stripped mucosal epithelia were placed into Ussing chambers and incubated with $5 \mathrm{mmol} / \mathrm{L}$ luminal glucose after equilibration. $\mathrm{Na}^{+} /$glucose absorption was measured by increases in short-circuit currents ( $\left.\mathrm{I}_{\mathrm{sc}}\right)$ and glucose fluxes ( 5 animals) were quantified by addition of ${ }^{3} \mathrm{H}$-glucose. Finally, phlorizin was added to the luminal buffer to inhibit SGLT1 activity.

Results: We found significant differences $(P<0.0001)$ between jejunum and ileum regarding basal $(0.4$ vs. $\left.-0.9 \mu \mathrm{eq} /\left(\mathrm{h}^{*} \mathrm{~cm}^{2}\right)\right)$ and glucose induced Isc values $\left(2.6\right.$ vs. $\left.5.4 \mu \mathrm{eq} /\left(\mathrm{h}^{*} \mathrm{~cm}^{2}\right)\right)$. The enhanced ileal transport capacity was also observable in glucose flux rates which were about fivefold higher than in the jejunum. Additionally, in ileal tissues the electrophysiological glucose response was significantly $(P<0.0001)$ more rapid in comparison with jejunal tissues, calculated by the periods that were needed to reach definite percentages of the maximal glucose response. Another significant discrepancy $(P<0.0001)$ between the intestinal segments could be seen with respect to SGLT1 inhibition. Addition of phlorizin reduced the glucose induced short-circuit current in the ileum to basal pre-glucose levels, while the glucose-induced increase was alleviated at only $80 \%$ in the jejunum. Analysis of phlorizin affected glucose flux rates showed that the ileum responded more sensitive with an estimated $\mathrm{K}_{\mathrm{i}} 50 \%$ of phlorizin of $0.012 \mathrm{mmol} / \mathrm{L}$ compared to $0.178 \mathrm{mmol} / \mathrm{L}$ in the jejunum.

Conclusions: Although the expression levels of SGLT1 are shown to be higher in the jejunum we observed a more effective glucose absorption in the ileum. The underlying molecular mechanisms for these surprising findings are still unknown, but the differential dynamics of the examined intestinal segments with regard to glucose response and phlorizin mediated inhibition indicate that the activity of SGLT1 might be influenced, directly or indirectly, by additional factors which are differently expressed at least in the porcine small intestine. 